\title{
The efficacy of chemotherapeutic drug combinations may be predicted by concordance of gene response to the single agents
}

\author{
ANDREW M. GRAVETT, JAYNE L. DENNIS, ANGUS G. DALGLEISH, JOHN COPIER and WAI M. LIU
}

Institute for Infection and Immunity, Department of Oncology, St. George's, University of London, London SW17 0RE, UK

Received September 19, 2019; Accepted July 10, 2020

DOI: 10.3892/ol.2020.12184

\begin{abstract}
Determining the expression of genes in response to different classes of chemotherapeutic drugs may allow for a better understanding as to which may be used effectively in combination. In the present study, the human colorectal cancer cell line HCT116 was cultured with equi-active concentrations of a series of anti-cancer agents. Gene expression profiles were then measured by whole-genome microarray. Although each drug induced a unique signature of gene expression in tumour cells, there were marked similarities between certain drugs, even in those from different classes. For example, the antimalarial agent artesunate and the platinum-containing alkylating agent, oxaliplatin, produced a very similar mRNA expression pattern in HCT116 cells with 14,000 genes being affected by the two drugs in the same way. Furthermore, the overall correlation of gene responses between two agents could predict whether their use in combination would lead to a greater or lesser effect on cell number, determined experimentally, than predicted by single agent experiments. The results indicated that even when working through different mechanisms, combining drugs that initiate a similar transcriptional response may constitute the best option for determining drug-combination strategies for the treatment of cancer.
\end{abstract}

\section{Introduction}

Combining chemotherapy drugs to treat cancer is a common approach used in an attempt to enhance treatment strategies, and to counteract the development of resistant cancer cells. For example, utilising drugs such as folinic acid, 5-fluorouracil (5-FU) and oxaliplatin (OXP) in combination that makes up the FOLFOX chemotherapy regimen has brought good efficacy in approaches to treat some colon cancers (1). Similarly, in breast cancer, a combination of cyclophosphamide, 5-FU, epirubicin or methotrexate are often used together. However,

Correspondence to: Dr Wai M. Liu, Institute for Infection and Immunity, Department of Oncology, St. George's, University of London, Cranmer Terrace, London SW17 0RE, UK

E-mail:wliu@sgul.ac.uk

Key words: chemotherapeutics, microarray, combinations, cancer the use of particular chemotherapy drugs in certain cancer are often selected based upon historical success of the single agent, and rationales for chemotherapy combinations are often nebulous and ill-defined (2).

Generally, drugs from different chemotherapeutic classes are partnered with one another because they may exhibit different mechanisms of action, have minimally overlapping spectra of toxicity and target discrete phases of the cell cycle (3). This is thought to increase efficacy of the treatments and reduces the probability of the tumour acquiring resistance. However, despite cancer treatment algorithms and a drive towards personalised medicine combination strategies can still lack a rigorous scientific rationale, and few have been assessed to establish optimal dose, schedule and delivery. Instead, a 'hit and hope' approach can be adopted, which does not necessarily lead to the most effective treatment for individual patients. Indeed, the vagaries of combination regimen when considered with the intrinsic diversity of tumours and sensitivities to differing treatments, means a more rational plan for the combination of chemotherapies is needed.

Identifying the drug combinations suitable for certain diseases requires a more careful understanding of the mechanisms of action for each drug involved in a regimen. It is only by understanding profoundly the effects of each that the best partners can be identified. However, and more bafflingly, it is also not enough to understand the effect of a drug as these typically are assessed in single-agent setting. Once drugs are combined, the profile and complexity of the interactions can alter the expected outcome. For example the use of monoclonal antibodies to neutralise cytotoxic T-lymphocyte associated antigen 4 (CTLA-4) can induce anticancer activity; however, this can cause a compensatory increase in the programmed-death 1 (PD-1) receptor, which can ultimately suppress the overall anticancer action. For this reason, the sequential use of CTLA-4 and PD-1 antagonists could overcome the resistance by using the former drug alone (4).

In addition to simply killing, chemotherapeutic drugs can interfere with intracellular processes rendering cells more susceptible to other treatments, be they chemo-, immuno- or radiotherapies (5-7). The effects on cell signalling can occur even when the chemotherapy is used at doses that do not have a great effect on cell number $(8,9)$. Attempts are being undertaken to assess the impact of the genetic landscape on drug efficacy (10). Knowledge of the signalling modulation and transcriptional changes instigated by each chemotherapy will 
allow for a more holistic approach to combination selection. Current thinking goes even further than this with 'precision' chemotherapy approaches being investigated, where even knowledge of the tumour's transcriptional background being taken into consideration when designing treatment regimens $(8,11,12)$.

Chemotherapy drugs can be divided into a number of different classes, including: Alkylating agents, such as oxaliplatin (OXP), that work by covalently cross-linking deoxyribonucleic acid (DNA) strands via their alkyl group; antimetabolites, such as gemcitabine (GEM), that block DNA replication; mitosis dysregulators, such as docetaxel (DOC), that stop cancer cells completing mitosis by interfering with proper microtubule function and others, such as the iMiDs, which target cereblon and have anti-proliferative, anti-inflammatory and anti-angiogenic properties $(13,14)$, and the artemisinins, which are anti-malarial drugs that have been reported to not only retard tumour cell growth in vitro but also be excellent combinatorial partners for other drugs $(15,16)$. Artemisinins initiate apoptosis in tumour cells through iron-catalyzed lysosomal activation and reactive oxygen species production $(17,18)$.

In this study the colorectal tumour cell line, HCT116, was treated with equi-active concentrations on OXP, GEM, DOC, the $\mathrm{iMiD}$ lenalidomide (LEN) and a semi-synthetic derivative of artemisinin, artesunate (ARS). A brief summary of each drug is shown in Table I. The effect of each drug on gene transcription was then measured by microarray and this was used to predict whether particular drugs would be exhibit synergistic or antagonistic characteristics when combined with one another to inhibit tumour cell growth.

\section{Materials and methods}

Tumour cell lines. The human colorectal cancer cell line HCT116 (Public Health England) was grown in complete DMEM medium (Sigma-Aldrich, Dorset, UK) supplemented with $10 \%$ foetal bovine serum (FBS) (Invitrogen, Paisley, UK), $2 \mathrm{mM}$ L-glutamine and $1 \%$ penicillin/streptomycin (both Sigma). Authentication of this cell line was performed by the service provider using the AmpFISTR Identifier Plus PCR amplification kit looking for the presence of $<10$ known loci for each cell line. Cells were incubated in a humidified atmosphere with $5 \% \mathrm{CO}_{2}$ in air at $37^{\circ} \mathrm{C}$. When approximately $75 \%$ confluency was reached, cells were harvested with trypsin (Sigma-Aldrich) prior to washing and reseeding at a lower cell density. Only cells with a passage number $<15$ were used in experiments.

Drugs. GEM, OXP, CPM, camptothecin (all from Sigma-Aldrich), artesunate (ARS) (St. George's Hospital Pharmacy) and LEN (Celgene Corp.) were reconstituted in phosphate-buffered saline (PBS) (Sigma), or in the case of LEN and DOC, dimethyl sulfoxide (DMSO) (Sigma-Aldrich), to create a top stock solution of $100 \mathrm{mM}$, which and stored at $-80^{\circ} \mathrm{C}$ long-term and $-20^{\circ} \mathrm{C}$ short-term (up to one month). Where necessary, all drugs were diluted in PBS. When adding drug to cell culture, volume and DMSO concentration were maintained for each condition, DMSO concentration was always $<0.01 \%$. Cells were allowed to adhere overnight before drugs were added at equi-active concentrations based on our previous work $(5,15)$. Final concentrations were: ARS $(1 \mu \mathrm{M})$, DOC (10 nM), GEM (100 nM), LEN $(1 \mu \mathrm{M})$, NAL $(1 \mu \mathrm{M})$ and OXP (500 nM). Tumour cells were then cultured for a further $48 \mathrm{~h}$, at which time, cell numbers were assessed by MTT. Additionally, the effect of treatment on the gene expression was assessed by isolating RNA from cells cultured with the drugs for $4 \mathrm{~h}$. These samples were processed for subsequent microarray analyses.

Illumina microarrays. RNA was isolated from control or HCT116 cells treated with ARS, DOC, GEM, LEN, NAL or OXP using the Qiagen mini-kit according to the instructions of the manufacturer. The concentration and quality of the resultant RNA was determined using NanoDrop and 2100 Bioanalyzer (Agilent Technologies). The RNA was found to be extremely pure with $\mathrm{OD}_{260 / 280}$ ratios measured by nanodrop $>1.90$ and electropherograms confirmed intact RNA. Microarrays were performed by Dr Jayne Dennis at the St. George's, University of London Biomics Centre. Biotinylated cRNA was generated from $100 \mathrm{ng}$ total RNA using the Illumina TotalPrep RNA Amplification Kit (Applied Biosystems; Thermo Fisher Scientific, Inc.) according to manufacturer's instructions. Equal amounts (750 ng) of cRNA were hybridised to the Illumina human HT12-v3 arrays for $18 \mathrm{~h}$. These arrays consisted of more than 48,000 probes covering RefSeq and UniGene annotated genes, and acquisitions were subsequently processed according to manufacturer's instructions before scanning on an Illumina BeadArray Reader. The image data were processed using default values in GenomeStudio v2009.1 with imputation of missing data, before loading onto GeneSpring v9.0 for data normalisation and filtering. Gene-ontology (GO)-enrichment analysis was also performed (19-21). Gene activity values represent the mean of three separate experiments that were used as scientific replicates i.e. each experiment represented a single well on the microarray chip.

Methylthiazoletetrazolium (MTT) assays. To study the effect of drugs on the number of viable cells, cells growing exponentially were added to 96 -well plates at a density of $3 \times 10^{4}$ cells $/ \mathrm{ml}$ in $180 \mu \mathrm{l}$ complete medium. After the cells had been allowed to adhere overnight, $20 \mu \mathrm{l}$ of drug stock solutions were added to the wells to the appropriate final concentration. The number of viable cells was measured at $48 \mathrm{~h}$ using a standard MTT-based assay without modifications. Briefly, MTT (Sigma-Aldrich) was added to each well to give a working concentration of $0.4 \mathrm{mg} / \mathrm{ml}$, and plates returned to $37^{\circ} \mathrm{C}$ for a further $\mathrm{h}$. After this time, the medium was aspirated off, $200 \mu 1 \mathrm{DMSO}$ added to each well and plates agitated gently for one minute before measuring optical density at $550 \mathrm{~nm}$ using a microplate reader (Dynex-MRX II; Dynex Technologies Ltd.). Each sample was run in triplicate and experiment was completed three times.

Statistical analysis of correlation and gene ontology analysis. $\mathrm{R}^{2}$ values and Spearman's Rank Correlation Coefficients were obtained using Microsoft Excel 2019 v1808. Gene ontology (GO) analysis was performed using Gene Ontology Resource (http://geneontology.org/). 
Table I. List of drugs used for microarray experiments.

\begin{tabular}{lll}
\hline Name & \multicolumn{1}{c}{ Class } & \multicolumn{1}{c}{ Target } \\
\hline Artesunate & Anti-malarial & Lysosomal Iron \\
Camptothecin & Topoisomerase Inhibitor & $\begin{array}{l}\text { Topoisomerase } \\
\text { Microtubules }\end{array}$ \\
Docetaxel & Mitosis inhibitor & DNA \\
Gemcitabine & Antimetabolite & Cereblon \\
Lenalidomide & Immune modulatory & Not known \\
Naltrexone & Other & DNA \\
Oxaliplatin & Alkylating agent & \\
\hline
\end{tabular}

\section{Results}

Each drug produced a specific signature of gene activation/inhibition in HCT116 cells. HCT116 cells treated with equi-active concentrations of various drugs were assessed by microarray. The 25 most upregulated and 25 most downregulated genes were then listed for each treatment and the change in those genes in response to the other drugs was then evaluated (Fig. 1). Genes with a $\geq 3$-fold mRNA expression increase after treatment are shown in green, graded to white which indicates no change. Genes with a $\geq 3$-fold mRNA decrease after treatment are shown in red, again graded to white which indicates no change. The left-hand panel shows the genes that were most strongly upregulated or downregulated in response to GEM and how those same genes were affected by the other treatments. OXP and CAM appeared to upregulate some of the same genes as GEM, as shown by the number of genes highlighted in green in the second lane $(\mathrm{O})$ and sixth lane $(\mathrm{C})$ in the top half of the panel. Genes that were strongly upregulated by GEM were not greatly affected by DOC, LEN and ARS, indicated by the pale grading of the genes in these lanes. Conversely, genes that were downregulated by GEM, shown in the bottom half of the left panel and coloured red were upregulated by DOC and consequently appear green. This discordance between GEM and DOC holds true when the analysis is performed on the genes most affected by DOC treatment (3rd panel on top row), where many of the genes that are most upregulated by treatment with DOC are downregulated by GEM. By analysing the data in this way it is possible to infer which treatments are more similar or more different with regards to their effect on gene transcription. The drugs DOC, LEN and ART seemed to produce a similar pattern of gene changes in HCT116 cells, as did GEM, CAM and OXP. The gene changes induced by naltrexone treatment did not appear to share many similarities with the other treatments.

The pattern of affected genes was similar between drugs with disparate mechanisms. To understand whether similarities in genetic profiles of different drugs could determine the combination benefit, HCT116 cells were cultured with equi-active concentrations of each of the drug, and RNA extracted after $4 \mathrm{~h}$ for gene microarray analyses. Changes in gene expression relative to the untreated control were then sorted for each drug, and the similarities in their patterns determined. Gene expression was termed 'altered by treatment' if the expression had changed $\pm 25 \%$ or more from control and otherwise regarded as unchanged. Gene lists were then judged against each other to determine how changes in the expression of the individual genes compared and contrasted following a particular treatment.

The effect of the treatment on each gene in the microarray was assessed and changes that were in agreement in terms of the direction of change, irrespective of magnitude, were defined as being concordant. For example, expression of the gene, aurora kinase A, was increased following treatment with GEM and CAM, decreased after DOC and NAL and unchanged in response to OXP, LEN and ART. Consequently, this gene was called concordant between GEM and CAM, discordant between GEM and DOC or NAL and mixed between GEM and the other treatment pairs. By using this method of analysis, pairs of drugs could be assessed for the extent of the concordance in genes. Results showed that ART and LEN had the greatest number of genes $(14,186)$ that were altered in a similar manner following treatment (Fig. 2A). Conversely, CAM and NAL had the fewest number of concordant genes (7343) and the most mixed (Fig. 2B) and discordant genes (Fig. 2C). As a larger proportion of concordant genes could be those unchanged after treatment, these were excluded in subsequent analysis where the percentage of concordant, discordant or mixed genes for each treatment pair is shown (Fig. 2D).

There were good correlations in the changes to certain gene families following treatment. The effect of each of the drugs on genes named on the Qiagen 'apoptosis gene list' was assessed. The changes were then ranked in order of the magnitude of change for each gene, and these ranked-lists compared with each other. For example, the $\mathrm{r}^{2}$-value was 0.664 for lenalidomide vs. docetaxel, which suggested a good positive correlation in apoptosis-genes affected by the drugs (Fig. 3A). Conversely, there was no correlation between the apoptosis-related genes affected by naltrexone and oxaliplatin $\left(\mathrm{r}^{2}=-0.022\right.$; Fig. 3B). A similar analysis using lists of genes involved in the cell cycle showed similarities and differences between the drugs; with lenalidomide and docetaxel being the drug pair displaying the highest correlation $\left(r^{2}=0.610\right.$; Fig. 3C) and naltrexone and lenalidomide displaying the least correlation $\left(\mathrm{r}^{2}=0.014\right.$; Fig. 3D). In addition to any effects on apoptosis or cell cycle genes, gene ontology enrichment analysis revealed which cellular processes were impacted by each treatment (Table II). Genes related to the DNA damage response, cellular stress, apoptosis and cell cycle were overrepresented in the 100 most affected genes after culturing cells with the DNA damaging chemotherapies such as CAM, GEM and OXP. Each of these drugs seemed to be involved in the activation or repression of numerous cellular processes. In contrast, DOC showed only enrichment in 'cell division' and LEN in no processes at all. That is not to say that LEN did not affect any cellular process, just that the drug did not affect any particular process disproportionately.

Combinational value correlated with gene concordance. MTT analysis was performed on HCT116 cells that had been cultured with the chemotherapeutic drugs either as single agents or as selected combinations for $48 \mathrm{~h}$, a representative example of data is shown in Fig. 4A. The difference between the expected effect on cell number by combining two drugs (generated by adding together of the results of the effect on cell 

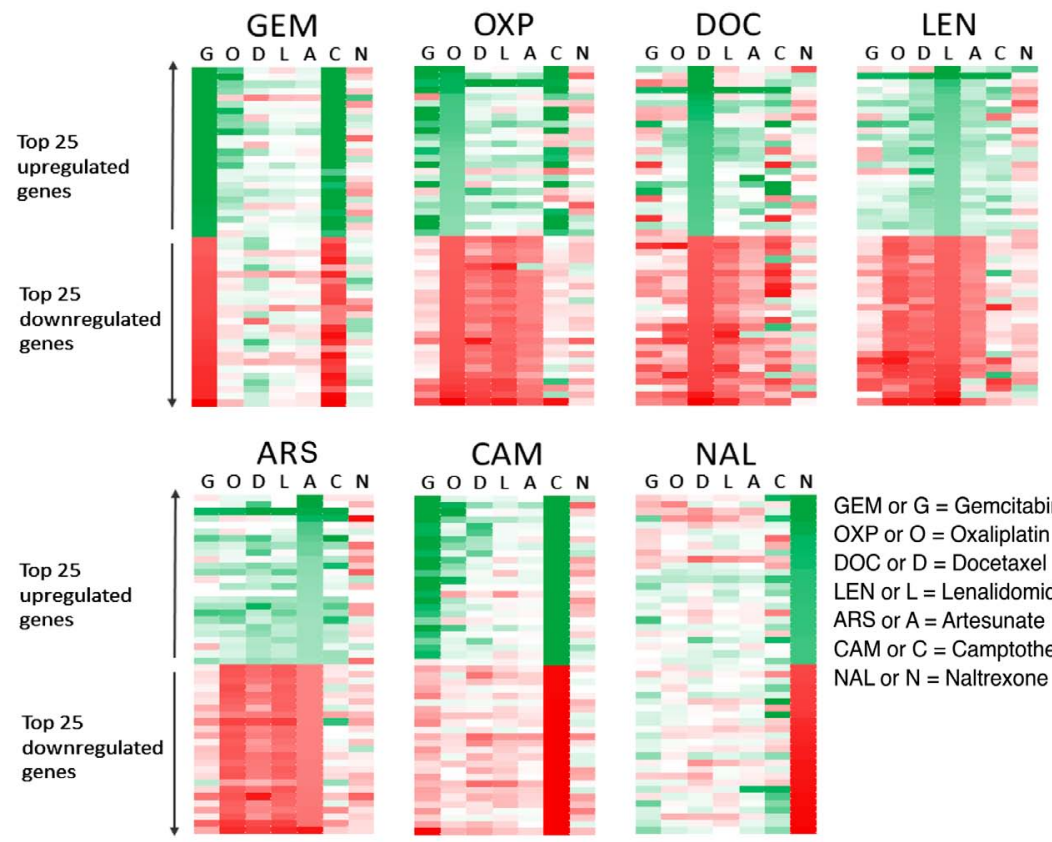

NAL

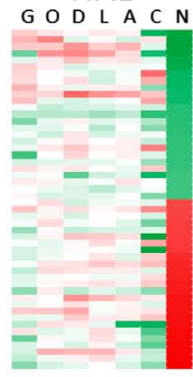

GEM or $\mathrm{G}=$ Gemcitabine OXP or $\mathrm{O}=$ Oxaliplatin $\mathrm{DOC}$ or $\mathrm{D}=$ Docetaxel LEN or $L=$ Lenalidomide ARS or $\mathrm{A}=$ Artesunate CAM or $\mathrm{C}=$ Camptothecin NAL or $\mathrm{N}=$ Naltrexone

Figure 1. Heat maps demonstrating the genes most affected by each drug and how this compared with other treatments. The 25 most upregulated and 25 most downregulated genes in response to each drug in microarray experiments were listed and then presented in terms of their response to other drugs. Colour grading indicates the magnitude of change from untreated controls: Green indicates $\geq 3$-fold increase, white indicates no change, red indicates $\geq 3$-fold decrease. Data taken from microarray experiments were repeated three times and used as a single replicate.
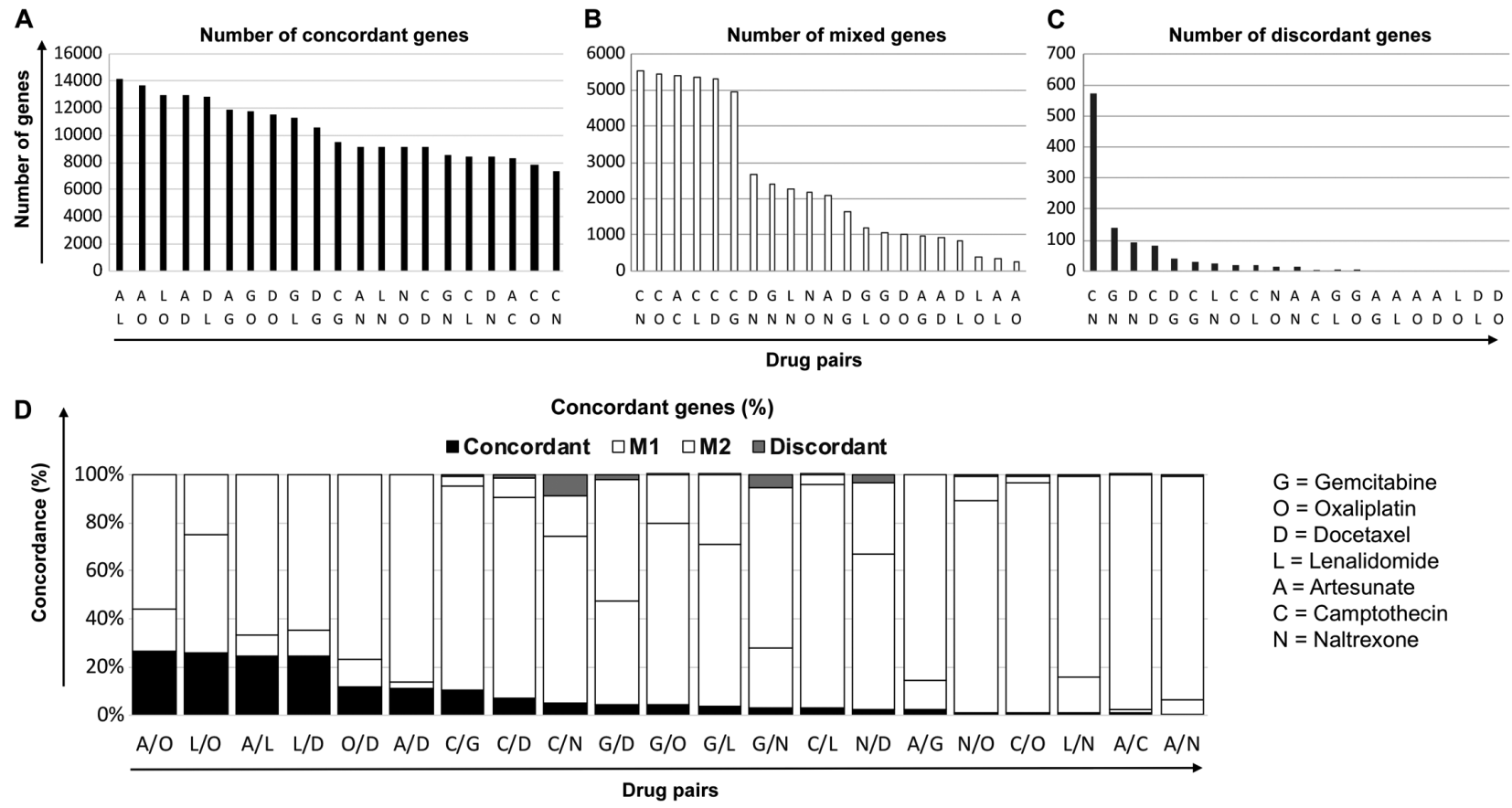

$\mathrm{G}=$ Gemcitabine

$\mathrm{O}=$ Oxaliplatin

$\mathrm{D}=$ Docetaxel

$\mathrm{L}=$ Lenalidomide

$A=$ Artesunate

$\mathrm{C}=$ Camptothecin

$\mathrm{N}=$ Naltrexone

Figure 2. Data form microarray experiments was used to compare drug pairs for concordance of gene responses. The cut-off used for a change in gene expression was a $20 \%$ increase or decrease from untreated controls. (A) The number of concordant genes for each drug pair is presented. Genes were termed concordant if they responded to both drugs in the same way. (B) The number of 'mixed' genes is presented. Mixed genes were defined as those which were altered in response to one drug but unchanged in response to the other. (C) The number of genes that were discordant between drug pairs is demonstrated. For example, increased by one drug and decreased by the other. (D) presents the percentage of genes that were concordant, mixed (M1 and M2) or discordant for each drug pair. Genes that were unchanged in response to both drugs of a drug pair were removed from analysis.

number of each single agent) was then compared to the actual result of combining the two drugs together. Using this analysis it was possible to find drug combinations that performed better or worse than the predicted effect by single-agent analysis (Fig. 4B). The combination of artesunate and lenalidomide performed the best, exceeding the predicted effect on cell number. The combinations of artesunate and docetaxel and artesunate and oxaliplatin also performed better than expected. The combination of lenalidomide and camptothecin performed worst and had a less than predicted effected on cell 
A

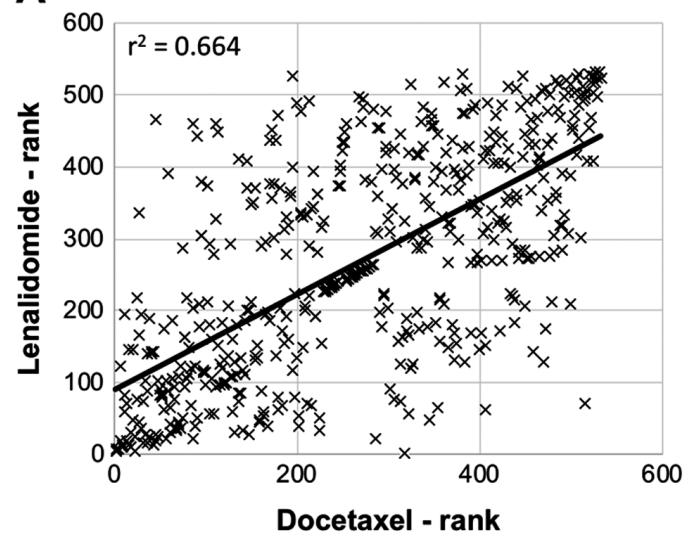

C

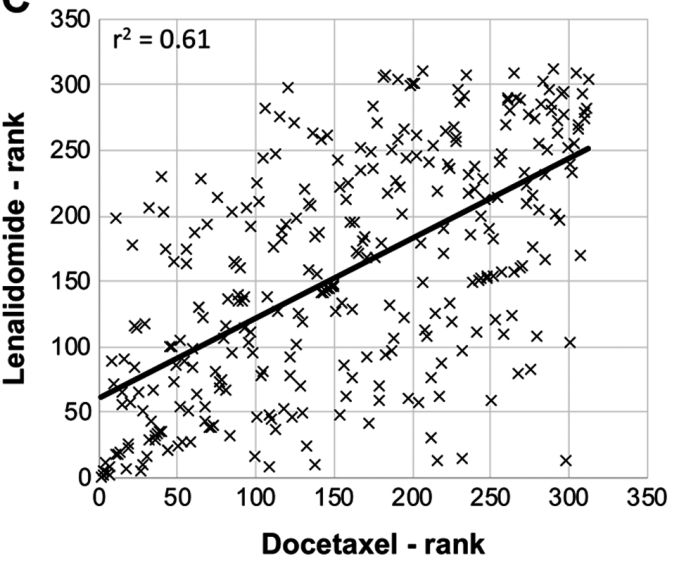

B

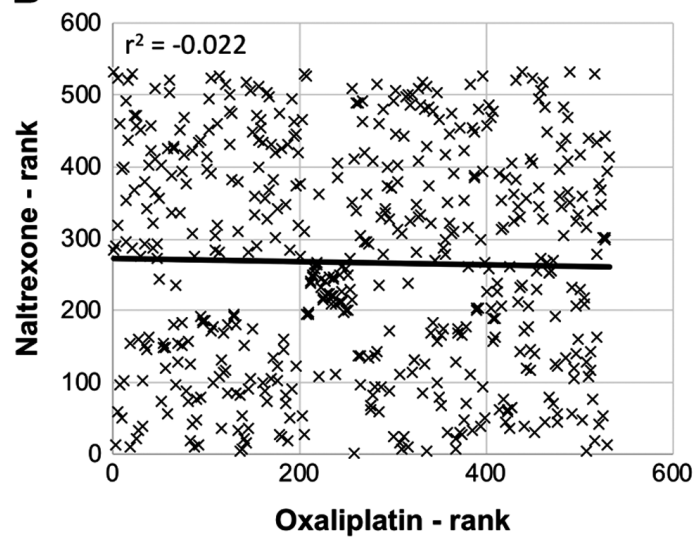

D

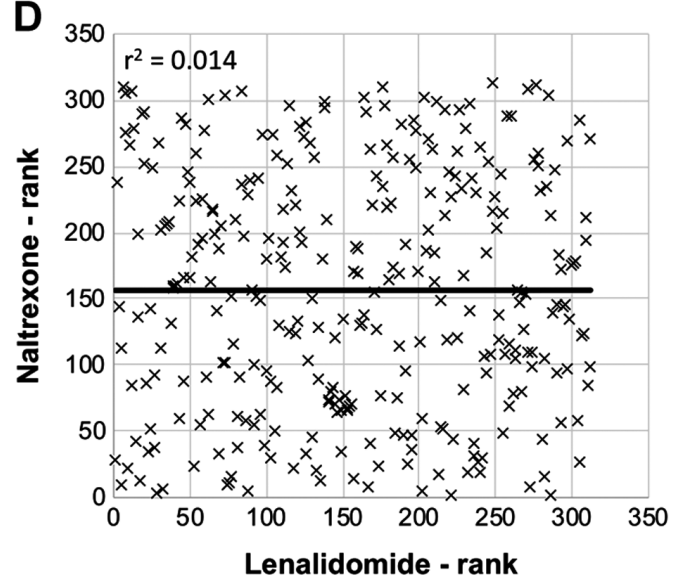

Figure 3. Apoptosis and cell cycle related genes were defined using Qiagen gene lists. Microarray data were then used to rank each gene in terms of its response to each drug. Graphs representing strongest and weakest correlations are presented. (A) There was a strong positive correlation between the way lenalidomide and docetaxel affected apoptosis genes. (B) There was no correlation between the way apoptosis genes were affected by naltrexone and oxaliplatin. (C) There was a strong positive correlation between the way lenalidomide and docetaxel affected cell cycle genes. (D) There was no correlation between the way apoptosis genes were affected by naltrexone and lenalidomide.

number suggesting antagonism between these two drugs at the concentrations used here. The combinations were ranked and then compared with the rankings of the drugs in terms of their transcriptional concordance, discordance, apoptosis response and cell cycle response (Fig. 4C). The best correlation occurred when MTT data was compared with overall gene concordance (Fig. 4D), suggesting that this was the best predictive measure for combinational efficiency.

\section{Discussion}

The current study compares the mRNA expression profiles of HCT116 tumour cells after they have been cultured with different chemotherapeutic agents. The tumour cells were cultured with suboptimal concentrations of different classes of chemotherapy and the response measured by whole-genome microarray. Despite the different targets and mechanisms of action of the drugs, there were some similarities in the pattern of gene activation/inhibition for some of the drugs. Moreover, drugs that displayed general concordance in gene signature were shown to exhibit synergistic traits when combined with one another.

A number of drugs have multiple mechanisms of action, and so deciding drug-partners in combination regimens simply on the basis of the principal mechanism of action may inadvertently miss opportunities. For example, we have shown naltrexone, which is a proficient antagonist of opioid receptors can also reduce the expression of the cyclin-dependent kinase inhibitor $\mathrm{p} 21^{\text {waf1 }}$ (22). This suggests it could be used in combination with other drugs that modify cell cycle functions. Previous studies have investigated the correlation between the mRNA expression patterns in tumour cells and; growth inhibition by agents (23), drug resistance $(24,25)$ and prognosis $(26)$, but these studies have usually focussed on untreated cells, and not attempted to predict combination value by comparing the mRNA expression profiles of tumour cells after culture with different classes of drug. In the current study, we specifically examined the gene signatures in one colon cancer cell line after treatment with a range of chemotherapy drugs, and analysed these profiles for patterns that could identify similarities in MOAs. Data pertaining to certain genes from cells treated with gemcitabine has been published in our previous papers $(5,7)$. Data pertaining to naltrexone has been published previously (30).

For some time now, the identification of genetic patterns in patients with certain types of cancer has been used as a prognostic indicator. Indeed, in some cases, establishing the genetic fingerprint of the a cancer can guide the type of treatment $(27,28)$. The current study differs from this more general approach by first identifying the genes that are changed following treatment. It is hoped that by matching these signatures, certain drugs that have similar genetic profiles 
Table II. The one hundred most affected genes (by fold change) from microarray data for each drug were used as gene sets for analysis using the GO Resource Enrichment Analysis Tool. This analysis determines which GO terms are over- or under-represented using annotations for these gene sets. The table shows significant shared GO Biological Process terms used to describe each set of genes. These data reveal which cellular mechanisms may be hit by each drug.

Drug GO Terms

Artesunate Lipid droplet organization; intrinsic apoptotic signaling pathway in response to endoplasmic reticulum stress; positive regulation of neuron apoptotic process; cellular protein localization; negative regulation of gene expression; regulation of cellular metabolic process; regulation of primary metabolic process; regulation of nitrogen compound metabolic process.

Camptothecin Positive regulation of extrinsic apoptotic signaling pathway via death domain receptors; intrinsic apoptotic signaling pathway in response to oxidative stress; DNA damage response, signal transduction by p53 class mediator resulting in cell cycle arrest; apoptotic mitochondrial changes; cellular response to UV; positive regulation of protein kinase B signalling; cellular response to starvation; positive regulation of protein kinase activity; regulation of cell population proliferation; regulation of response to stress.

Docetaxel Cell division.

Gemcitabine Mitotic chromosome movement towards spindle pole; meiotic sister chromatid cohesion, centromeric; spindle assembly involved in meiosis; mitotic metaphase plate congression; mitotic spindle assembly checkpoint; DNA damage response, signal transduction by p53 class mediator resulting in cell cycle arrest; cellular response to amino acid starvation; positive regulation of cyclin-dependent protein kinase activity; regulation of cyclin-dependent protein serine/threonine kinase activity; regulation of mitotic spindle organization; centromere complex assembly; mitotic spindle organization; regulation of cytokinesis; G2/M transition of mitotic cell cycle; cellular response to UV; anaphase-promoting complex-dependent catabolic process; cell division; intrinsic apoptotic signaling pathway; regulation of G2/M transition of mitotic cell cycle; positive regulation of cellular catabolic process; DNA conformation change; positive regulation of cell population proliferation; regulation of apoptotic process.

Lenalidomide N/A

Oxaliplatin Response to corticosterone; DNA damage response, signal transduction by p53 class mediator resulting in cell cycle arrest; positive regulation of cysteine-type endopeptidase activity involved in apoptotic process; intrinsic apoptotic signaling pathway; transcription initiation from RNA polymerase II promoter; cellular response to extracellular stimulus; regulation of neuron death; response to radiation; regulation of apoptotic signaling pathway; cell development; regulation of gene expression.

GO, gene ontology.

may be supportive of one another. A similar study examining the RNAi signatures of mammalian cell death genes highlighted the importance of mechanism in drug combination and revealed, for example, that the mapping of 17AAG and taxol in the same region in the principal component analysis space, supported the prediction that 17AAG would reinforce a taxol-like action (29).

Our previous work has shown that the same agent can have diverse effects on genes when used at different concentrations (30). At high concentrations, a number of drugs will cause a large amount of cell death and have a catastrophic effect on gene translation, so for this reason, we cultured cells with drugs at sub-optimal concentrations. Additionally, microarrays were performed on RNA extracted from cells after $4 \mathrm{~h}$ of culture with drug to ensure that the secondary/tertiary effects of the drugs and non-specific effects on gene expression were minimised and so the primary fingerprint of effect could be determined for each drug. Artesunate, camptothecin, docetaxel, gemcitabine, lenalidomide, naltrexone and oxaliplatin each initiated a particular gene response when cultured with HCT116 cells at suboptimal concentrations. However, despite the varying molecular targets and mechanisms of action for the drugs, there were similarities between the patterns of genes hits by certain pairs of drugs. For example, there was a remarkable concordance in the mRNA expression of tumour cells cultured with artesunate and lenalidomide. This despite the disparate nature of the mechanisms of action and molecular targets of these drugs, artesunate potentially working through iron-catalysed alkylating cytotoxic free radicals in tumours and lenalidomide targeting cereblon (31). Conversely, drugs such as gemcitabine and docetaxel, and, naltrexone and camptothecin, had very different microarray responses, in many cases genes that were upregulated by one drug would be downregulated by the other. This kind of reciprocal response may be expected for drugs that, for example, target different points on the cell cycle, and although GO analysis (Table II) of the 50 most upregulated and the 50 most downregulated genes suggested that gemcitabine, although multifaceted, affects $\mathrm{G}_{2} / \mathrm{M}$ transition, while docetaxel affects cell division only, there were cell cycle protein-specific changes that highlighted 
A

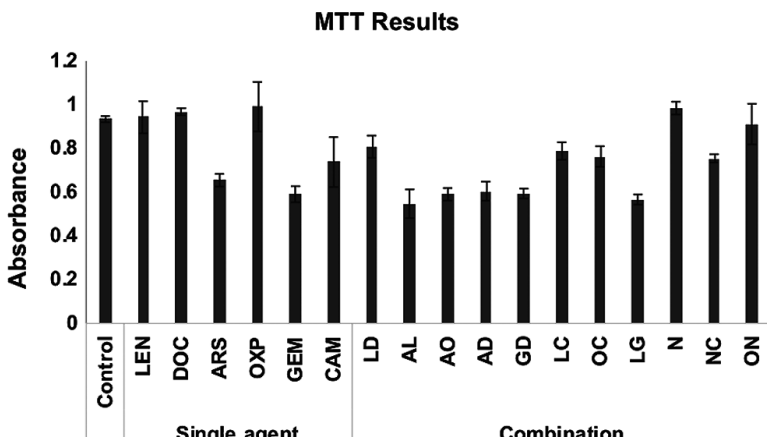

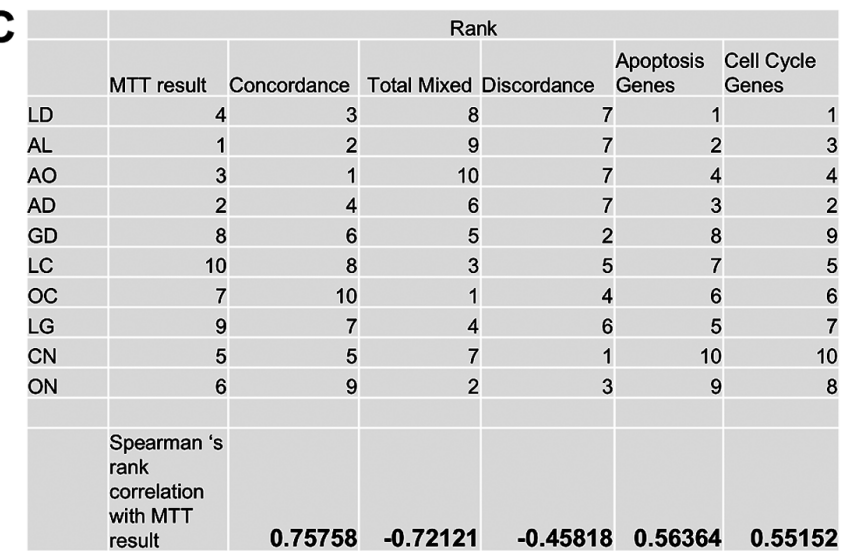

B Combinations - Difference from predicted
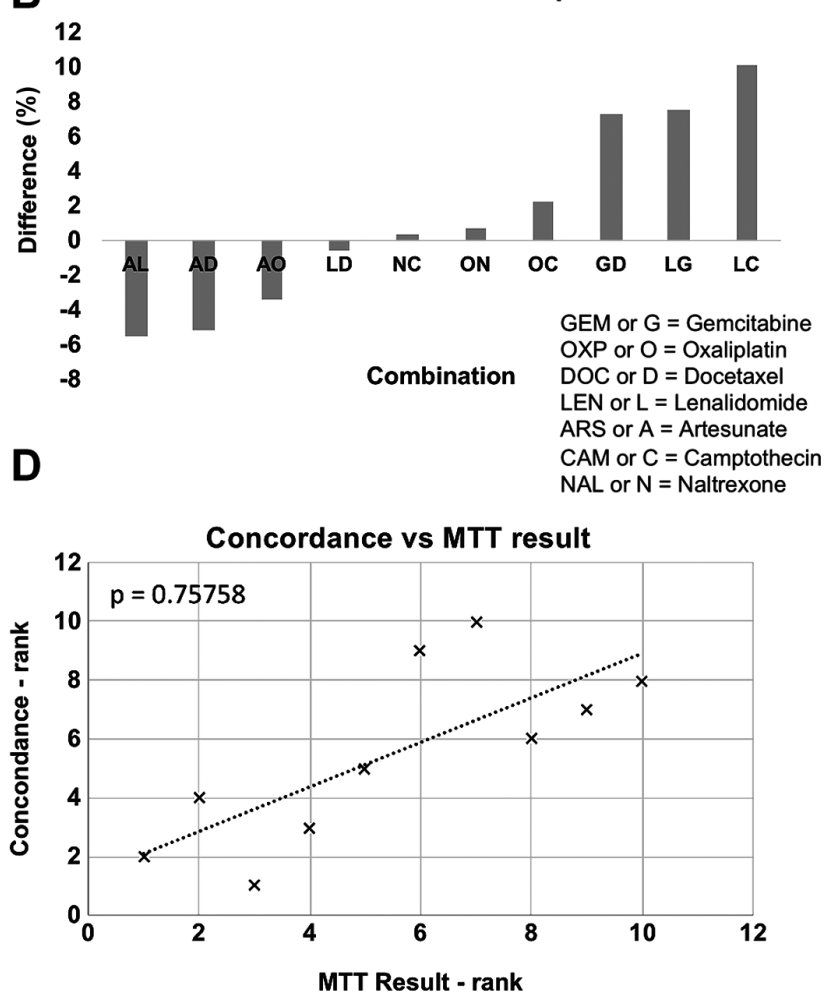

Figure 4. Combination effectiveness was determined by a MTT assay correlated with gene expression concordance. (A) Representative MTT experiment demonstrating raw absorbance data from a single MTT assay corresponding to the number of metabolically viable HCT116 cells after various treatments and combinations. (B) The efficiency of each drug combination. Each drug combination is presented on the $\mathrm{x}$-axis while columns represent percentage difference from the expected cell number reduction if the drug combinations were additive. Combinations where the percentage difference was positive or negative exhibit a detrimental or beneficial effect of the combination on cell number. Data are presented as the mean difference from three separate experiments. (C) Chemotherapy combinations were ranked in terms of their ability to reduce cell number better than expected. These rankings were then correlated with those for gene concordance, discordance, apoptosis gene response and cell cycle gene response using Spearman's rank correlation. (D) The combination efficiency of two drugs, as defined as a better than expected result from MTT assays, most strongly correlated with gene concordance between the two drugs.

this difference. For example, gemcitabine upregulated cyclin E and marginally downregulated cyclin A, while for docetaxel the opposite was true, cyclin $\mathrm{E}$ being reduced, and cyclin $\mathrm{A}$ being upregulated.

This kind of inverse response on gene transcription suggests that the drugs, when combined, would neutralise each other, abrogating the response of both in terms of gene changes and cytotoxicity/cytostasis. What may help to indicate an efficacious combination is by examining the effects of the drugs on genes regulating key processes such as apoptosis and the cell cycle. By focusing on apoptosis-associated genes, it was revealed that docetaxel and lenalidomide had the most similar response i.e. both drugs showed a similar pattern of response in genes associated with apoptosis. This despite the fact that docetaxel is generally viewed as a potent cytotoxic agent, whilst lenalidomide is generally thought of as non-toxic. Parallel to this, docetaxel and lenalidomide produced a similar response in cell cycle-associated genes in HCT116 cells again signifying that although docetaxel and lenalidomide have different potencies and mechanisms of action, the end result of their use in HCT116 tumour cells is transcriptionally similar.

Cells contain numerous sensors that perceive damage and then relay this to machinery involved with cellular repair, death or senescence (32). The present study shows that even when using vastly differing chemotherapies with diverse molecular targets and MOAs, there can be an overlap in the
mRNA-transcripts that are affected. Moreover, correlation between chemotherapy and gene response cannot necessarily be predicted by the similarity of drugs. For example, gemcitabine and oxaliplatin both block DNA replication by inserting into DNA strands; however, in terms of the general mRNA response, only $4.2 \%$ of affected genes were 'concordant'. Conversely, artesunate and oxaliplatin supposedly have totally different MOAs and yet $26.5 \%$ of genes were impacted by the individual drugs in the same way.

Upon determining the gene response to the various chemotherapies, we next wanted to know whether these could be predictive of the combination effect when using the drugs concomitantly. Intuitively, combining drugs with discordant gene signatures would lead to a situation where the effects imparted by the individual drugs would negate the actions of the other, thereby neutralising the response. However, combining drugs that were not discordant could potentially be beneficial as they could, for example, better activate desired gene responses in a cooperative manner (concordance) or activate more than one desired response (mixed). Indeed, using MTT analysis it was found that additivity between drugs correlated most strongly to overall gene concordance between drugs. That is to say, drugs with similarities in their transcriptional responses combined better to reduce cell number, compared to those with transcriptional effects that were less similar. 
The fundamental rationale for combination therapy in an oncology setting is to optimise overall action by using drugs that work together to target tumour masses that contain cells that are often heterogeneous (33). This heterogeneity means that the cell types can be diverse enough to prevent drugs with focussed MOAs lacking the activity to elicit a therapeutic effect. Therefore, mixing drugs to allow a more diverse range of action could be beneficial. Similarly, it could be argued that the over-reliance of a drug concentrating on one specific target could allow resistance to the drug to develop more readily (34). However, even drugs with very similar MOAs can have very different responses, such as those reported with platinum derivatives in cisplatin-resistant cell lines (35). Despite this, most clinical data and scientific dogma would suggest that combining drugs with identical MOAs would not be best practise and this idea is still maintained in the present study, which suggests not combining similar drugs but combining very different drugs that have a similarity of response at the transcriptional level. Further investigation of this effect using more cell lines and different chemotherapies is needed to determine whether this theory holds true.

Due to the nature of the study the combination that performed best in terms of reduction of cell number may not have been the combination that reduced cell number to the largest extent, as combinations were ranked in terms of performance compared to predicted performance, not which combination reduced cell number the most. This study is also limited by the fact that only one concentration of each of the drugs was used. We can say with some certainty that titration of the drugs to different concentrations will produce a different pattern of transcriptional response and it may also be that changing the concentrations will also influence whether the drug combinations retain their beneficial or detrimental effects on tumour cell number. A further confounding factor not even touched upon in this study is the importance of drug sequence when using more than one agent $(22,36)$. For these reasons, these data can only be used as a guide as to which drugs may combine well with one another and it is essential that further studies include an investigation of drug dose.

The present study suggests that an important factor in the strategic combination of chemotherapies may be that there is some correlation in the transcriptional response induced by the chemotherapeutics that are being combined. That is not to say that the drugs should not have different targets and mechanisms but the present data suggest that the outcome of this should be a similar pattern of gene expression to allow the best chance for combinational synergy.

\section{Acknowledgements}

Not applicable.

\section{Funding}

The present study was funded by the Institute for Cancer Vaccines and Immunotherapy.

\section{Availability of data and materials}

The datasets used and/or analysed during the current study are available from the corresponding author on reasonable request. Microarray data is available in the GEO respository accession number GSE122985.

\section{Authors' contributions}

AMG and WML designed the current study, acquired and analysed the data and wrote the manuscript. JLD performed the experiments and acquisition of data. AGD and JC designed the current study, interpreted data and wrote the manuscript. All authors read and approved the final manuscript.

\section{Ethics approval and consent to participate}

Not applicable.

\section{Patient consent for publication}

Not applicable.

\section{Competing interests}

AMG is presently funded by Celgene Corporation. WML is presently funded by LDN Pharma Limited. The remaining authors declare that they have no competing interests.

\section{References}

1. O'Connell MJ: Current status of adjuvant therapy for colorectal cancer. Oncology (Williston Park) 18: 751-755; discussion 755-758, 2004.

2. Almendro V, Ametller E, García-Recio S, Collazo O, Casas I, Augé JM, Maurel J and Gascón P: The role of MMP7 and its cross-talk with the FAS/FASL system during the acquisition of chemoresistance to oxaliplatin. PLoS One 4: e4728, 2009.

3. Yardley DA: Drug resistance and the role of combination chemotherapy in improving patient outcomes. Int J Breast Cancer 2013: $137414,2013$.

4. Buchbinder EI and Desai A: CTLA-4 and PD-1 pathways: Similarities, differences, and implications of their inhibition. Am J Clin Oncol 39: 98-106, 2016.

5. Gravett AM, Dalgleish AG and Copier J: In vitro culture with gemcitabine augments death receptor and NKG2D ligand expression on tumour cells. Sci Rep 9: 1544, 2019.

6. Scott KA, Dalgleish AG and Liu WM: The combination of cannabidiol and $\Delta 9$-tetrahydrocannabinol enhances the anticancer effects of radiation in an orthotopic murine glioma model. Mol Cancer Ther 13: 2955-2967, 2014.

7. Gravett AM, Trautwein N, Stevanović S, Dalgleish AG and Copier J: Gemcitabine alters the proteasome composition and immunopeptidome of tumour cells. Oncoimmunology 7: e1438107, 2018.

8. Nars MS and Kaneno R: Immunomodulatory effects of low dose chemotherapy and perspectives of its combination with immunotherapy. Int J Cancer 132: 2471-2478, 2013.

9. Tsimberidou AM, Eggermont AM and Schilsky RL: Precision cancer medicine: The future is now, only better. Am Soc Clin Oncol Educ Book 2014: 61-69, 2014.

10. Hu HM, Zhao X, Kaushik S, Robillard L, Barthelet A, Lin KK, Shah KN, Simmons AD, Raponi M, Harding TC and Bandyopadhyay S: A quantitative chemotherapy genetic interaction map reveals factors associated with PARP inhibitor resistance. Cell Rep 23: 918-929, 2018.

11. McKenna MT, Weis JA, Brock A, Quaranta V and Yankeelov TE: Precision medicine with imprecise therapy: Computational modeling for chemotherapy in breast cancer. Transl Oncol 11: 732-742, 2018.

12. Deng $X$ and Nakamura Y: Cancer precision medicine: From cancer screening to drug selection and personalized immunotherapy. Trends Pharmacol Sci 38: 15-24, 2017.

13. Pan B and Lentzsch S: The application and biology of immunomodulatory drugs (IMiDs) in cancer. Pharmacol Ther 136: 56-68, 2012. 
14. Hideshima T, Chauhan D, Shima Y, Raje N, Davies FE, Tai YT, Treon SP, Lin B, Schlossman RL, Richardson P, et al: Thalidomide and its analogs overcome drug resistance of human multiple myeloma cells to conventional therapy. Blood 96: 2943-2950, 2000.

15. Liu WM, Gravett AM and Dalgleish AG: The antimalarial agent artesunate possesses anticancer properties that can be enhanced by combination strategies. Int J Cancer 128: 1471-1480, 2011.

16. Gravett AM, Liu WM, Krishna S, Chan WC, Haynes RK, Wilson NL and Dalgleish AG: In vitro study of the anti-cancer effects of artemisone alone or in combination with other chemotherapeutic agents. Cancer Chemother Pharmacol 67: 569-577, 2011.

17. Hamacher-Brady A, Stein HA, Turschner S, Toegel I, Mora R, Jennewein N, Efferth T, Eils R and Brady NR: Artesunate activates mitochondrial apoptosis in breast cancer cells via iron-catalyzed lysosomal reactive oxygen species production. J Biol Chem 286: 6587-6601, 2011.

18. Yang ND, Tan SH, Ng S, Shi Y, Zhou J, Tan KS, Wong WS and Shen HM: Artesunate induces cell death in human cancer cells via enhancing lysosomal function and lysosomal degradation of ferritin. J Biol Chem 289: 33425-33441, 2014.

19. Ashburner M, Ball CA, Blake JA, Botstein D, Butler H, Cherry JM, Davis AP, Dolinski K, Dwight SS, Eppig JT, et al: Gene ontology: Tool for the unification of biology. The gene ontology consortium. Nat Genet 25: 25-29, 2000.

20. The Gene Ontology Consortium: The gene ontology resource: 20 Years and still GOing strong. Nucleic Acids Res 47: D330-D338, 2019.

21. Mi H, Huang X, Muruganujan A, Tang H, Mills C, Kang D and Thomas PD: PANTHER version 11: Expanded annotation data from gene ontology and reactome pathways, and data analysis tool enhancements. Nucleic Acids Res 45: D183-D189, 2017.

22. Scott KA, Dalgleish AG and Liu WM: Anticancer effects of phytocannabinoids used with chemotherapy in leukaemia cells can be improved by altering the sequence of their administration. Int J Oncol 51: 369-377, 2017.

23. Wallqvist A, Rabow AA, Shoemaker RH, Sausville EA and Covell DG: Establishing connections between microarray expression data and chemotherapeutic cancer pharmacology. Mol Cancer Ther 1: 311-320, 2002.

24. Tseng AH, Chung FH, Lee HC, Wu LC, Chen $\mathrm{CH}$ and Su LJ: Microarray analysis and establishment of drug screening platform using 5-fluorouracil resistance HCT116 colon cancer cells. Genomic Med Biomarkers Health Sci 4: 21-27, 2012.
25. Lønning PE, Sørlie T, Perou CM, Brown PO, Botstein D and Børresen-Dale AL: Microarrays in primary breast cancer-lessons from chemotherapy studies. Endocr Relat Cancer 8: 259-263, 2001.

26. Kao KJ, Chang KM, Hsu HC and Huang AT: Correlation of microarray-based breast cancer molecular subtypes and clinical outcomes: Implications for treatment optimization. BMC Cancer 11: 143, 2011.

27. Kamel HF and Al-Amodi HS: Exploitation of gene expression and cancer biomarkers in paving the path to era of personalized medicine. Genomics Proteomics Bioinformatics 15: 220-235, 2017.

28. Wang TH and Chao A: Microarray analysis of gene expression of cancer to guide the use of chemotherapeutics. Taiwan J Obstet Gynecol 46: 222-229, 2007.

29. Pritchard JR, Bruno PM, Gilbert LA, Capron KL, Lauffenburger DA, Hemann MT: Defining principles of combination drug mechanisms of action. Proc Natl Acad Sci USA 110: E170-E179, 2013.

30. Liu WM, Scott KA, Dennis JL, Kaminska E, Levett AJ and Dalgleish AG: Naltrexone at low doses upregulates a unique gene expression not seen with normal doses: Implications for its use in cancer therapy. Int J Oncol 49: 793-802, 2016.

31. Fink EC and Ebert BL: The novel mechanism of lenalidomide activity. Blood 126: 2366-2369, 2015.

32. Jackson SP and Bartek J: The DNA-damage response in human biology and disease. Nature 461: 1071-1078, 2009.

33. Liu WM: Enhancing the cytotoxic activity of novel targeted therapies-is there a role for a combinatorial approach? Curr Clin Pharmacol 3: 108-117, 2008.

34. Housman G, Byler S, Heerboth S, Lapinska K, Longacre M, Snyder N and Sarkar S: Drug resistance in cancer: An overview. Cancers (Basel) 6: 1769-1792, 2014.

35. Rixe O, Ortuzar W, Alvarez M, Parker R, Reed E, Paull K and Fojo T: Oxaliplatin, tetraplatin, cisplatin, and carboplatin: Spectrum of activity in drug-resistant cell lines and in the cell lines of the national cancer institute's anticancer drug screen panel. Biochem Pharmacol 52: 1855-1865, 1996.

36. Shah MA and Schwartz GK: The relevance of drug sequence in combination chemotherapy. Drug Resist Updat 3: 335-356, 2000.

(i) $($ This work is licensed under a Creative Commons Attribution-NonCommercial-NoDerivatives 4.0 International (CC BY-NC-ND 4.0) License. 